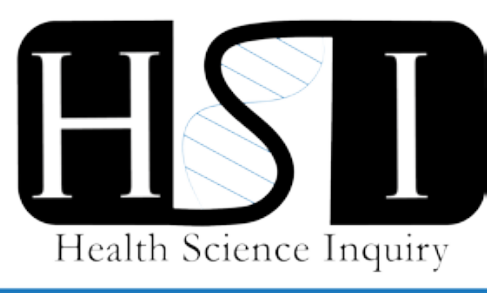

\title{
Stand up for your health: excessive sedentary behaviour as a modifiable risk factor for chronic disease
}

\author{
Yoah Sui ${ }^{1}$, Anna Rudkovska ${ }^{2}$ \\ ${ }^{1}$ Department of Kinesiology, University of Western Ontario; ${ }^{2}$ Department of Health \& Rehabilitation Sciences, \\ University of Western Ontario
}

Within daily life, there are few more ubiquitous behaviours than sitting. Excessive sitting is a form of sedentary behaviour (SB), which is defined as any waking behaviour in a seated, lying, or reclining position with an energy expenditure of 1.5 metabolic equivalents (i.e., 1.5 times the resting energy expenditure [1]), and has been consistently linked to an increased risk for all-cause mortality and numerous chronic diseases [2]. While SB does not include behaviours such as standing or sleeping, nearly every activity of daily life involves or encourages sitting: transportation, occupations, leisure-time, and household activities. Notably, SB is distinct from physical activity (PA); it is not simply the absence of it or the lack thereof. Rather, SB is a unique contributor to chronic disease risk, independent of PA levels. A recent review by Ekelund and colleagues [3] suggests that high levels of moderate-intensity PA (i.e., 60-75 min/day) can attenuate the detrimental effects of SB; however, these levels of activity are not easily attainable or feasible for population health, with only 1 in 5 Canadians meeting PA guidelines of $150 \mathrm{~min} /$ week [4].

Unlike other modifiable health behaviours-such as smoking or PA-SB is an invisible behaviour, in that engagement in it is typically not a conscious or cognitive choice. The excessive accumulation of SB is partially due to a built-up environment; however, factors such as occupation (e.g., manual labour vs. desk-based), education, and societal norms [5,6] all contribute to the average Canadian sitting for 10.8 hours/day [7]. This statistic is especially concerning when the dose-dependent nature of $S B$ is considered: the more an individual engages in $\mathrm{SB}$, the greater their risk of developing chronic health conditions.

Although the field of SB research is young, numerous reviews and meta-analyses have been published in the last decade, consistently and independently linking excessive $\mathrm{SB}$ to numerous chronic diseases. Notably, a systematic review by Rezende and colleagues [2] summarizes 27 systematic reviews that assess the association between $\mathrm{SB}$ and health outcomes. The authors report that SB is associated with all-cause mortality and cardiovascular mortality, independent of PA and body mass index (BMI), with some reviews reporting as much as a $49 \%$ increase in all-cause mortality between individuals who were sedentary for $<4$ hours/day and those who were sedentary for $>9$ hours/day. The same review by Rezende and colleagues reports a positive association between increased SB and risk for cardiovascular disease (5-15\%), cardiovascular events (5-17\%), type 2 diabetes (20-112\%), metabolic syndrome $(73 \%)$, and an obesogenic effect in youth [2]. There is also emerging evidence that eludes to an association between excessive SB and poor mental health [8].

It is hypothesized that a mechanism for the association between SB and chronic disease risk derives from low levels of lipoprotein lipase (LPL) following both acute and chronic exposure to SB [9], which are associated with an increased risk of cardiovascular disease. SB is also thought to affect carbohydrate metabolism through modifying muscle glucose transporter (GLUT) protein content, which negatively impacts insulin sensitivity and oxidative capacity. It should be noted that these hypothesized mechanisms are inferred from animal models, spinal cord injury studies, and bedrest studies [9]. Establishing the exact mechanism(s) of action of SB and the associated health effects still requires further investigation.

The risk for chronic disease is further amplified through the positive relationship between SB and other healthcompromising behaviours, including smoking, alcohol consumption, decreased leisure-time, physical activity, and diet [5]. While the causality of some of these behaviours is still debated, these negative health behaviours compound the risk for chronic diseases, such as obesity, cardiovascular disease, and diabetes.

Although the exact mechanism(s) for the relationship between SB and chronic diseases remain unknown, pragmatic solutions for preventing and attenuating the risks associated with excessive SB are simple, costeffective, and efficacious. A recent meta-analysis examined 
the effectiveness of breaks from SB on metabolic markers, and found that taking short, frequent, light-intensity PA breaks (e.g., walking) from bouts of sitting significantly improved blood glucose and insulin levels [10]. Breaks of moderate-vigorous PA were also more effective in reducing blood glucose and insulin levels than an equivalent singlebout of moderate-vigorous PA, highlighting the distinct benefit derived from taking multiple breaks compared to a single one [10].

Aside from the health benefits of breaking up prolonged $\mathrm{SB}$, the accessibility, simplicity, and cost associated with a SB intervention are ideal. Reducing overall SB by substituting it with standing and/or light physical activity is a simple, effective, and practically free solution that can be done by almost anyone at almost any time.

In sum, excessive SB negatively impacts health through increased risk of several chronic diseases, independent of PA levels. Breaking up bouts of SB with light-intensity $\mathrm{PA}$ or reducing overall $\mathrm{SB}$ are simple and effective ways for anyone to attenuate the unique risk associated with it.

\section{References}

[1] Tremblay, M. S., Aubert, S., Barnes, J. D., Saunders, T. J., Carson, V., Latimer-Cheung, A. E., et al. (2017) Sedentary Behavior Research Network (SBRN) Terminology Consensus Project process and outcome. Int J Behav Nutr Phys Act, 14 (1), 75. Available from: http://ijbnpa.biomedcentral.com/articles/10.1186/s129 66-017-0525-8

[2] de Rezende, L. F. M., Rodrigues Lopes, M., Rey-López, J. P., Matsudo, V. K. R., do Carmo Luiz, O. (2014) Sedentary Behavior and Health Outcomes: An Overview of Systematic Reviews. PLoS One, 9 (8), e105620. Available from: http://dx.plos.org/10.1371/journal. pone.0105620

[3] Ekelund, U., Steene-Johannessen, J., Brown, W. J., Fagerland, M. W., Owen, N., Powell, K. E., et al. (2018) Does physical activity attenuate, or even eliminate, the detrimental association of sitting time with mortality? A harmonised meta-analysis of data from more than 1 million men and women. The Lancet, 388 (10051), 13021310. Available from: http://dx.doi.org/10.1016/S0140-6736(16)30370-1

[4] Directly measured physical activity of Canadian adults, 2012 and 2013. Statistics Canada, 2015. Available from: https://www.statcan.gc.ca/pub/82625-x/2015001/article/14135-eng.htm

[5] O'Donoghue, G., Perchoux, C., Mensah, K., Lakerveld, J., van der Ploeg, H., Bernaards, C., et al., (2016) A systematic review of correlates of sedentary behaviour in adults aged 1865 years: a socio-ecological approach. BMC Public Health. 16 (1), 163. Available from: http://www.biomedcentral.com/1471-2458/16/163

[6] Owen, N., Sugiyama, T., Eakin, E. E., Gardiner, P. A., Tremblay, M. S., Sallis, J. F. (2011) Adults' sedentary behavior: Determinants and interventions. Am J Prev Med, 41 (2), 189196. Available from: http://dx.doi.org/10.1016/j.amepre.2011.05.013

[7] Carson, V., Wong, S. L., Winkler, E., Healy, G. N., Colley, R. C., Tremblay, M. S. (2014) Patterns of sedentary time and cardiometabolic risk among Canadian adults. Prev Med (Baltim), 65 (23), 2327. Available from: http://linkinghub.elsevier.com/retrieve/pii/S009174351 4001285

[8] Biddle, S. J. H., Bandelow, S. (2017) Sedentary behaviour and psychological well-being. In: Sedentary behavior and health: Concepts, evidence, assessment and intervention. Human Kinetics, 2017.

[9] Tremblay, M. S., Colley, R. C., Saunders, T. J., Healy, G. N., Owen, N. (2010) Physiological and health implications of a sedentary lifestyle. Appl Physiol Nutr Metab, 35 (6), 725740. Available from: http://www.nrcresearchpress.com/doi/abs/10.1139/H1 0-079

[10] Chastin, S. F. M., Egerton, T., Leask, C., Stamatakis, E. (2015) Meta-analysis of the relationship between breaks in sedentary behavior and cardiometabolic health. Obesity, 23 (9), 18001810. 

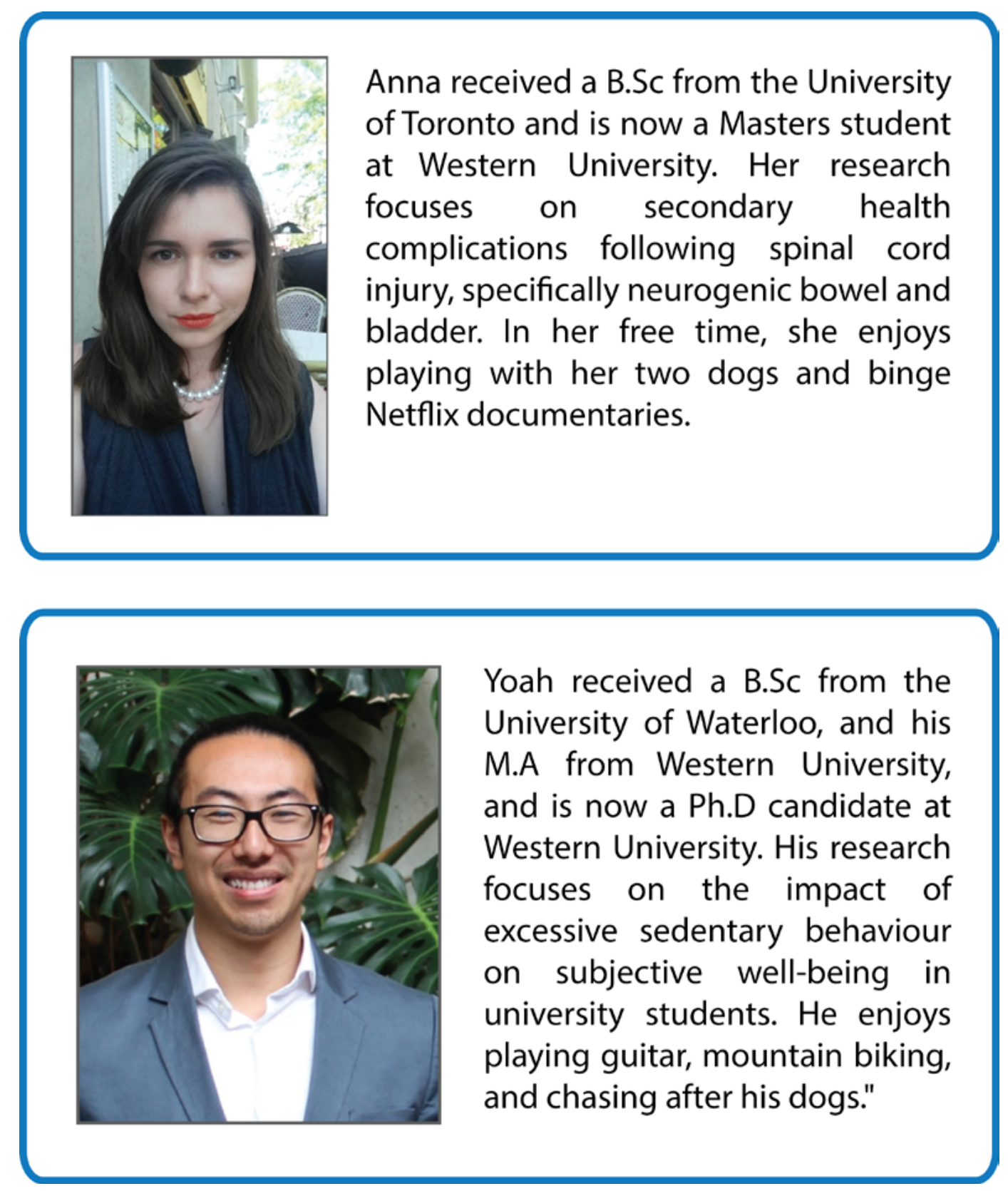